\title{
Ultra-Low Power CMOS RF Mixer for Wireless Sensor Networks Application: A Review
}

\author{
S.A.Z Murad ${ }^{1, *}$, Muhammad M. Ramli ${ }^{1}$, A. Azizan ${ }^{1}$, M.N.M Yasin ${ }^{1}$, and I.S Ishak ${ }^{1}$ \\ ${ }^{1}$ School of Microelectronic Engineering, University Malaysia Perlis, Pauh Putra Campus, 02600 \\ Arau, Perlis, Malaysia Sciences
}

\begin{abstract}
This paper reviews ultra-low power CMOS RF mixer for wireless sensor networks (WSN) application. The study elaborates several types of mixer, mixer's performance parameters, the mixer's topologies and also the design technique to improve the mixer performance. The previously published works are compared and the best topology for low power mixer is discussed.
\end{abstract}

\section{Introduction}

Wireless sensor networks (WSN) are self-organizing network system which contains a large number of sensors using wireless communication technology [1-2]. In general, the WSN almost designed for data collecting, processing and transmitting in a wide range of applications such as military and national security, home automation, environmental monitoring, industrial control and so on. Brief in [3] the WSN is significant in military and national security because it produces an efficient system where it has a self-organizing and fault tolerance characteristics mostly in military command, control, intelligence, surveillance, reconnaissance and targeting systems. For home automation, as a rapid demand on advance technology, the WSN is able to provide a smart sensor nodes and actuators by burying the sensors in appliances like vacuum cleaners, refrigerators and micro-wave ovens [4]. These sensor nodes inside the devices is working through interactions with each other and with the external network via the Internet or Satellite [3] and it allows the end users to manage home devices easily either in local or remote [4]. In addition, the WSN also a well-used sensors in the environmental monitoring like in ecosystems monitoring, pollution monitoring (air, water, soil, noise and etc.) and forest exploration; irrigation systems; meteorological; agriculture and also medical applications $[3,5-6]$. Fig. 1 shows the example of an overall system architecture supporting WSN applications which consist of RF transceiver and baseband processor.

Classically, the RF transceiver is built from several components such as low pass filter (LPF), mixer, power amplifier (PA), low noise amplifier (LNA) and voltage control oscillator (VCO). A mixer is an important component in a radio transceiver whose function is to translate the signal frequency to a higher or lower spectrum commonly generated by

*Corresponding author: sohiful@unimap.edu.my 
the multiplication of two signals [8]. Furthermore, the details of the mixer are discussed as section $\mathrm{f}^{*}$

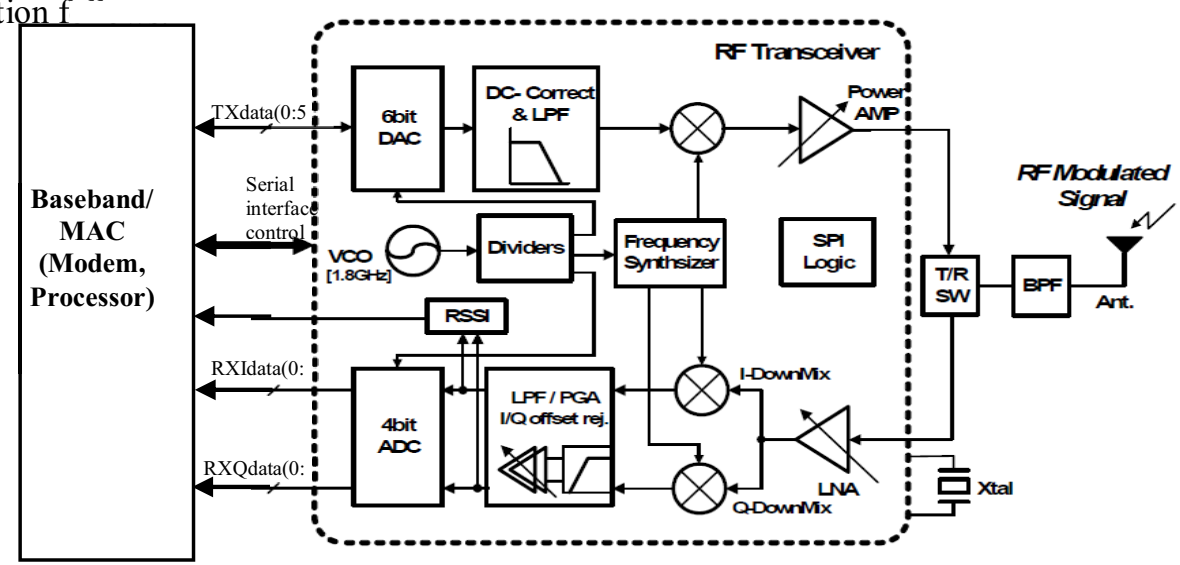

Fig. 1. Caption Example of an overall system architecture supporting WSN applications [7].

\section{Mixer's Topology}

The mixer circuit comprises a different topology which is suitable for a low power transmitters and receivers system. Based on previous researchers work there are three structures of mixer topologies which are a Gilbert-cell mixer, a bulk-driven mixer and a folded cascode mixer (FCM).

\subsection{Gilbert-cell mixer}

A Gilbert-cell is a conventional mixer whose frequently uses and most appropriate topology in designing of the active RF Mixer compared to other mixer's topologies. The Gilbert-cell topology [1,9] presents a several advantages such as attained a high linearity as well as conversion gain at low voltage operation. Besides that, this topology also has a good local oscillator (LO) to radio frequency (RF) and local oscillator (LO) to intermediate frequency (IF) port isolation in order to release the DC offset problems. Unfortunately, this structure is generated a large current that will affected by the noise of the mixer. The Gilbert-cell is includes of two different structures which are single-balance Gilbert-cell and doublebalance Gilbert-cell such illustrated in Fig. 2. A single-balance mixer is a simple structure that proposed a moderate conversion gain and linearity compared to the double-balanced mixer which provided a high linearity and increases the conversion gain of the circuit system. Besides that, the double-balanced mixer also can improve the inherent isolation between an input port as well as an output port. Nevertheless, this topology produces a higher insertion loss but it can be gained by applying the source inductance structure [11]. 


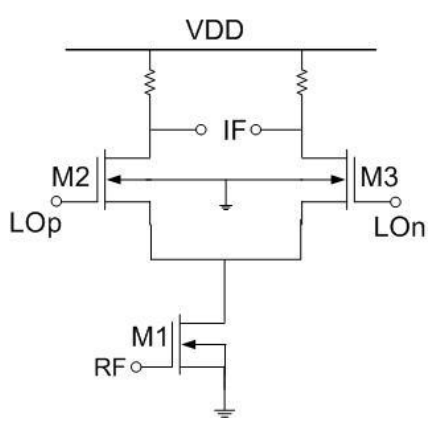

(a)

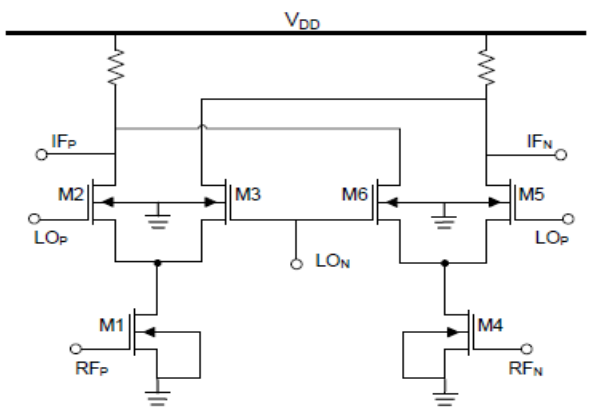

(b)

Fig. 2. Conventional Gilbert-cell topology: (a) Single-balanced mixer, (b) Double-balanced mixer [11].

\subsection{Bulk-driven mixer}

In order to reduce the DC power consumption, the bulk-driven has been applied in designing the mixer circuit. This topology is formed by using a bulk terminal of transistor to be used as a controller terminal as well as RF and LO signals. However, this technique applies to depends on the target of performance before the bulk is connected to the required terminals [12]. Nevertheless, this topology also can produce a low voltage supply since the topology provided less stacked transistors. Fig. 3 illustrates the examples of bulk-driven topology that often used in mixer circuit design.

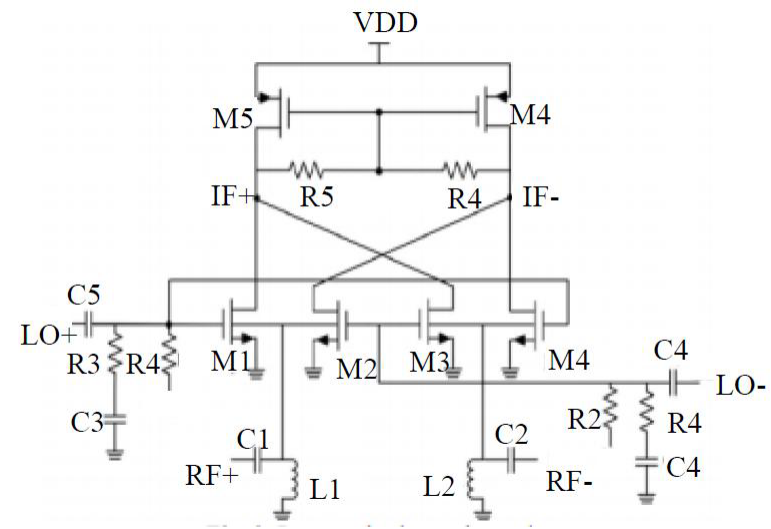

Fig. 3. Bulk-driven topology in conventional Gilbert cell mixer [13].

\subsection{Folded cascode mixer}

As reported in [22] the folded cascode mixer has been utilized for low power and lowvoltage mixer. Ideally, the folded cascode topology is designed as Fig. 4 where the positive and negative RF input signals are differently injected into transconductance of a differential amplifier of M1 and M2. Meanwhile the IF output signals are generated from the switching stage and load stage. Essentially, the folded cascade obtained low supply voltage due to the 
fold configuration which has depressed the system's requirement for dc voltage [22]. Although, this fold configuration gives also a good noise performance because the current flow through the switching stage is smaller. However, the folded cascade topology is not easy in verifying the linearity of mixer circuit, but this matter can be solved by increasing the transconductance (gm) stage biasing current.

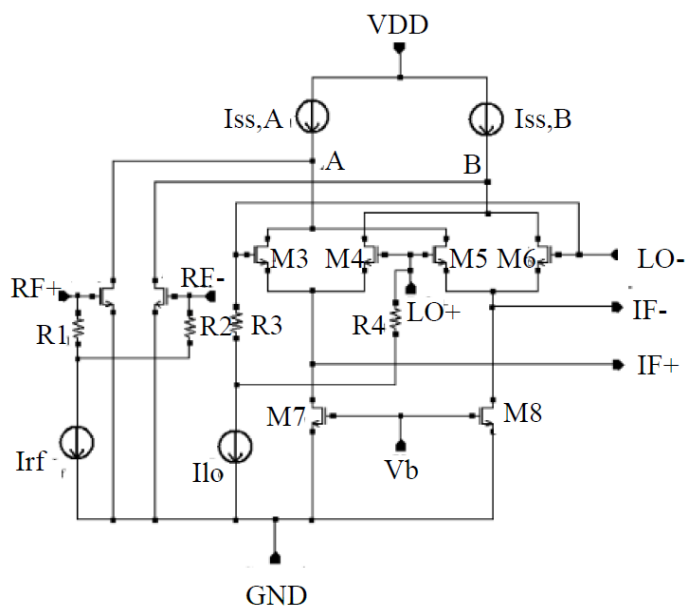

Fig. 4. Bulk- Folded cascode mixer topology [22].

\section{Circuit design technique}

A high-performances of the mixer circuit indicated by the parameters of the linearity as well as the conversion gain of the mixer core structure as depicted in Fig. 5. The mixer core consists of three major stages which are transconductance (gm) stage, switching stage and load stage. Therefore, there are several design techniques that can be applied in the enhancement of the linearity and also the conversion gain performances.

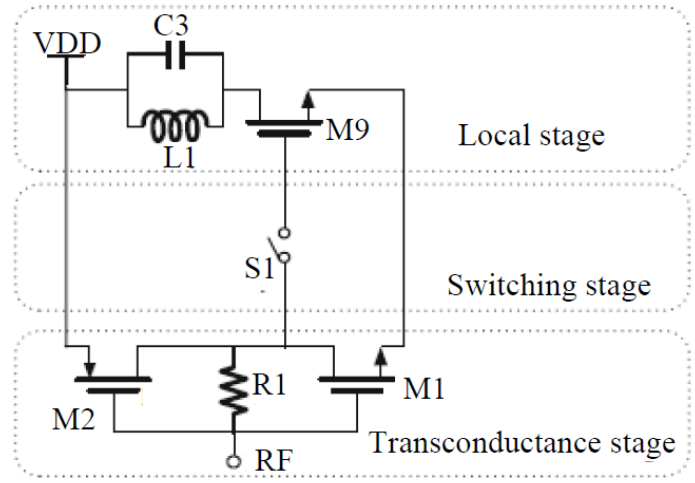

Fig. 5. Mixer core [23]. 


\subsection{Self-bias}

A self-bias technique proposed two advantages which are improving the conversion gain (CG) and also the linearity (IIP3) in the RF transconductance stage (gm). This technique is operated by the transistors alternately switched themselves using the IF input signals [15].

\subsection{Current-reuse current-mirror}

Current-reuse current-mirror technique is widely used consecutively to improve the gain and to reduce the power consumption of circuit systems. Fig. 6 shows the current-reuse current-mirror that has been modified from the traditional NMOS current-mirror structure. In brief, this technique can be reused the dissipated current at any stage to other stage because the total number of stacked transistor is not reduced same as the supply voltage $[14,16]$.
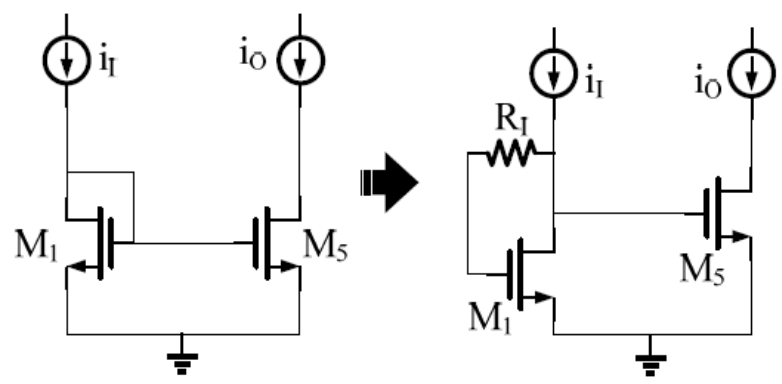

Fig. 6. The modification of traditional NMOS current-mirror to proposed current-mirror for currentreuse technique [2].

\subsection{Current bleeding}

The current bleeding technique is shown in Fig. 7 is particularly works as the injected current source as well as a part of the driving amplifier [2]. Commonly, this technique is constructed by connecting the RF input from the transconductance (gm) stage to the input of the bleeding stage. As reported in [17] the current is branched out in controlling the amount of a DC current through the transconductance (gm) stage and the switching stage independently. During this technique, the conversion gain of the mixer can be increase since this technique allowed a small amount of current flow through the switching stage besides reducing the gate-source capacitance $(\mathrm{Cgs})$ of the switching transistor. Hence, this technique also improved the switching efficiency. 


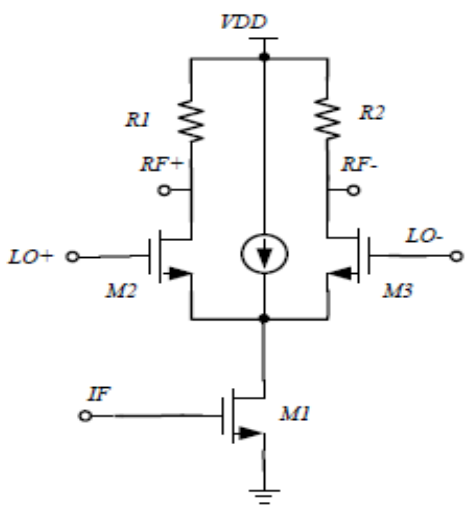

(a) Traditional

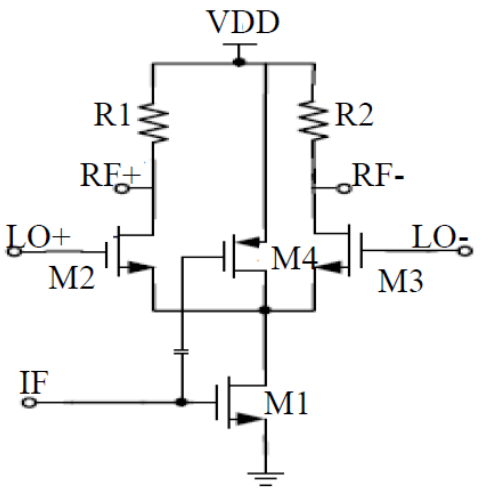

(b) PMOS

Fig. 7. Current bleeding [5].

\section{Summary of finding}

Figures A high-performances and efficient, ultra-low power CMOS RF mixer becomes a crucial circuit block in transceiver system especially for wireless sensor networks (WSN) applications. The characteristics of low power are important parameter for mixer performance, however at the same time the high gain and good linearity also need be considered. Thus, to provide good performances of these parameters, the suitable mixer topology must be chosen. A Gilbert-cell topology with double-balanced structure proposed a several advantages which include a high conversion gain as well as high linearity. Besides that, this topology also can enhance the inherent isolation between an inputs and output port of the mixer. In contrast, this topology consumes large currents and it will affect to the noise of the mixer. However, this drawback can be improved by applying the current bleeding technique because this technique can reduce the currents in the mixer circuit system through the switching stage. On the other hand, the current-reuse current-mirror can also be used because it can dissipate the currents at any stage and reduced the power consumption in the mixer circuit.

Table 1 summarizes the performances of CMOS RF mixers that have been done in the previous works. The proposed mixers [1-2],[21-22] indicates a low power of RF mixer which is around $0.25 \mathrm{~mW}$ to $1.36 \mathrm{~mW}$ by using a low voltage operation of $1.0 \mathrm{~V}$ and also the current-reuse current-mirror technique. Due to the characteristics of conversion gain (CG) and linearity (IIP3), the proposed works of [18] and [24] consumes larger CG compared to the other works because the mixer circuit has been designed by using the current bleeding technique. Besides that, as shows in the work [14] and [18], the mixer circuit provides a good linearity (IIP3) and produces lowest noise figure (NF) amongst to the other works since the self-bias technique and has been used in designing the mixer circuit. 
Table 1. The performance comparison of RF CMOS mixers.

\begin{tabular}{|c|c|c|c|c|c|c|c|}
\hline Reference & {$[\mathbf{1 9}]$} & {$[\mathbf{2 0}]$} & {$[\mathbf{2 1}]$} & {$[\mathbf{2 2}]$} & {$[\mathbf{1}]$} & {$[\mathbf{2}]$} & {$[\mathbf{1 0}]$} \\
\hline Technology $(\mu \mathrm{m})$ & 0.18 & 0.13 & 0.18 & 0.18 & 0.18 & 0.18 & 0.18 \\
\hline $\mathrm{V}_{\mathrm{DD}}(\mathrm{V})$ & 1.8 & $1.0-1.2$ & 1.2 & 1.0 & 1.0 & 1.0 & 1.0 \\
\hline Frequency $(\mathrm{GHz})$ & 2.4 & 2.4 & 2.4 & 2.4 & 2.4 & 2.4 & 2.4 \\
\hline Conversion gain $(\mathrm{dB})$ & 33 & 1 & 27.8 & 9.0 & 6.37 & 5.0 & 10.5 \\
\hline Noise figure $(\mathrm{dB})$ & 4.5 & 5.4 & 5.4 & 13.9 & 15.3 & $\mathrm{n} / \mathrm{a}$ & $\mathrm{n} / \mathrm{a}$ \\
\hline \begin{tabular}{c} 
IIP3 (dBm) \\
\hline P1dB (dBm)
\end{tabular} & -12 & $12-40$ & -23 & 2.2 & $\mathrm{n} / \mathrm{a}$ & -0.7 & 2.63 \\
\hline \begin{tabular}{c} 
Current consumes $(\mathrm{mA})$ \\
\hline $\begin{array}{c}\text { Power consumption } \\
(\mathrm{mW})\end{array}$
\end{tabular} & 5.8 & $\mathrm{n} / \mathrm{a}$ & -30 & -7.6 & -10 & -11 & 10.3 \\
\hline
\end{tabular}

\section{Conclusion}

A review of an ultra-low power CMOS RF Mixer for wireless sensor network application has been discussed. The most popular topology is the Gilbert-cell mixer due to high isolation between the input port and output port and high conversion gain. The CMOS RF mixer can be designed in a low power by using a current-reused because it can dissipate the currents at any stage, thus reduce the power consumption.

This work was financially supported by the Fundamental Research Grant Scheme (9003-00387).

\section{References}

1. W. Chenjian, L. Zhiqun, S. Ge., J. of Semiconductor, 35, 1 (2014).

2. S.A.Z. Murad, M.M. Shahimin, R.K. Pokharel, H. Hanaya, K. Yoshida, Micro. Opt. Technol. Lett., 54, 923 (2012).

3. I.F. Akyildiz, W. Su, Y. Sankarasubramaniam, E. Cayirci., Computer Networks, 38, 393 (2002).

4. E.M. Petriu, N.D. Georganas, D.C. Petriu, D. Makrakis, V.Z. Groza., IEEE Instrum. Meas. Mag., 3, 31 (2000).

5. S.A.Z. Murad, M.M Shahimin, M.N. Md Isa, R.C. Ismail, N. Ahmad, M.N.M Yasin, Micro. Opt. Technol. Lett., 57, 427 (2015).

6. M.L. Oliveira, P.C Rodrigues. J. Commun., 6, 143 (2011).

7. J. Gutierrez, J.F Villa-Medina, A. Nieto, M.A Porta. IEEE Trans. Instrum. Meas., 63, 166 (2014).

8. Information on httt://www.intechopen.com/books/wireless-sensor-network/ 
9. M. Voltti, T. Koivisto, E. Tiiliharju., 18th European Conference on Circuit Theory and Design, (2007).

10. R. Mahmou, K. Faitah, International Journal of electronics and Communications (AEU), 68, 883 (2014).

11. J.D. Chen, Z-M Lin, J.S Row, Analog Integr. Circ. S, 62, 301 (2010).

12. Allen Gibson. Design and Simulation of CMOS Active Mixers, (University of Central Florida Orlando, Florida, 2011).

13. E. Abiri, M.R. Salehi, M. Bahrebar. International Conference on Power Electronics, Systems and Applications, (2012)

14. S. Dan, Z. Xiaolin. Chinese Journal of Aeronautics, 23, 198 (2010)

15. L. Zhao, C. Wang. Springer Journal, Wireless Pers Communication, 80, 277 (2015).

16. M.Kim, J. Lee, T. Yun, Electronic Letters, 48, 1476 (2012)

17. S. He, C.E Saavedra, IEEE Trans. Micro. Theory Techn., 60, 311 (2012)

18. G.H. Tan, R.M. Sidek, M.M. Isa, IEEE Asia Pacific Conference on Circuits and Systems (APCCAS), (2014)

19. C. Choi, J. Choi, I. Nam., IEEE 9th International Conference on ASIC (ASICON), (2011)

20. K. Ghosal, T. Anand, V. Chaturvedi, B. Amrutur. 19th IEEE International Conference on Electronics, Circuits and Systems (ICECS), (2012)

21. C.H. Li, C.Y. Hsu, C.N. Kuo. IEEE 54th International Midwest Symposium on Circuits and Systems (MWSCAS), (2011).

22. M. Li, Z. Li, C. Wu, M. Zhang, J. Cao, Z. Wang, Adv. Mat. Res., 760, 526 (2013).

23. G. Sun, Z. Li, C.Wu, M.Zhang, J.Cao, Z. Wang, Adv. Mat. Res., 760, 516 (2013). 